\author{
Military Technical College \\ Kobry El-Kobbah, \\ Cairo, Egypt
}

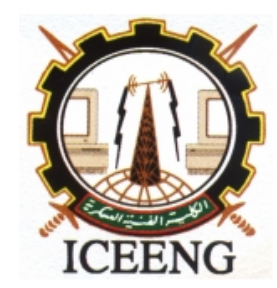

\author{
$7^{\text {th }}$ International Conference \\ on Electrical Engineering \\ ICEENG 2010
}

\title{
Compact and Tunable Matching of High Impedance Loads at microwave frequencies using metamaterials
}

\author{
By \\ Mahmoud Abdelrahman Abdalla* \\ Z. $\mathrm{Hu}^{* *}$ \\ A. G. Sobih*
}

\section{$\underline{\text { Abstract: }}$}

This paper presents, the analysis and design performance of a high impedance tunable left handed coplanar waveguide transformer designed on a ferrite substrate. The performance of the proposed transformer is explained analytically. Then numerical electromagnetic full wave simulations verify its theoretical behavior. The results illustrates that the proposed transformer can match a $200 \Omega$ load and a $50 \Omega$ line. The whole operating bandwidth can be tuned by changing the applied DC magnetic bias. The center frequency changes from $2.8 \mathrm{GHz}$ to $3.4 \mathrm{GHz}$. The proposed transformer length is only $2.4 \mathrm{~mm}$ which is approximately $78 \%$ reduction in conventional $\mathrm{RH}$ transformer length operates within the same frequency band. Moreover, the proposed transformer utilizes a horizontally magnetized ferrite substrate yielding a small demagnetization field and hence smaller required DC magnetic bias compared to microstrip configuration.

\section{Keywords:}

metamaterials, left handed, microwave circuits, ferrite

* Egyptian Armed Forces

** School of Electrical and Electronic Engineering, University of Manchester, Manchester, UK 


\section{Introduction:}

Left handed (LH) metamaterials are artificially constructed materials having electromagnetic properties that do not exist in nature. LH metamaterials have simultaneously negative permittivity and permeability. The LH metamaterials have been realized in planar version using transmission line loaded with complementary SRR (CSRR)/capacitive gap pairs [1]. A transmission line (TL) periodically loaded with series capacitors and shunt inductors can also exhibit left handed properties [2], [3]. Different LH coplanar waveguide (CPW) TL different models have been proposed [4]. Many novel planar microwave components LH elements have been introduced making use of the unique properties of the LH structures [5], [6]. Quarter wavelength LH TL transformer has a compact size and arbitrary designed frequency which can be utilized in many microwave applications.

Due to the dispersive properties of ferrite medium properties [7], a tunable and nonreciprocal LH TL has been demonstrated using ferrite substrate in both microstrip and CPW configurations [8], [9].

In this paper, we introduce a model of a compact and tunable quarter wavelength LH TL transformer with high load impedance. The current proposed model transformer is deigned to match a load of $200 \Omega$ to a $50 \Omega$ line. The proposed transformer is implemented using ferrite LH CPW TL. The left handed configuration is formed using only one unit cell constructed using shunt meandered line inductor and series air gap capacitors. The proposed transformer is studied analytically and its performance has been verified numerically.

\section{Theory}

The conventional quarter wavelength transmission line transformer achieves an electrical length of $\Phi=-\pi / 2$ at the operating frequency. Consequently, its length is equal to $\lambda / 4$ where $\lambda$ is guided wavelength.

One of the unique properties of LH TLs is its nonlinear phase properties due to the contribution of its left handed loading elements and its right handed parasitic elements. Thus, it can achieve $\pi / 2$ phase shift condition at frequency which is arbitrary and it is not necessary to be corresponding to its quarter wavelength frequency [5]. Instead, the design operating frequency is controlled by both loading and parasitic elements such that the $\pi / 2$ operating point is within the LH passband.

The phase shift along a ferrite LH TL of length 1 and its characteristic impedance can be 
redefined in terms of its medium parameters, the ferrite relative permittivity $\varepsilon_{\mathrm{f}}$ and the relative permeability $\mathrm{f},[9]$ as

$$
\begin{aligned}
& \phi=\omega l \sqrt{\left(\mu_{o} \mu_{f}-\frac{1}{\omega^{2} C_{L}^{\prime}}\right)\left(\varepsilon_{o} \varepsilon_{f}-\frac{1}{\omega^{2} L_{L}^{\prime}}\right)} \\
& Z_{L H}=\sqrt{\frac{L_{L}^{\prime}}{C_{L}^{\prime}}} \sqrt{\frac{1-\omega^{2} \mu_{o} \mu_{f} C_{L}^{\prime}}{1-\omega^{2} \varepsilon_{o} \varepsilon_{f} L_{L}^{\prime}}}
\end{aligned}
$$

where $\mathrm{L}_{\mathrm{L}}^{\prime}$, and $\mathrm{C}_{\mathrm{L}}^{\prime}$ represent the product of the loading inductance and capacitance with the left handed section length. Since the ferrite relative permeability $(\mathrm{f})$ is dispersive quantity, then both these two electrical parameters, phase shift and characteristic impedance, will be dispersive according to the applied DC magnetic bias. Thus, a tunable performance of this ferrite LH TL is expected when it acts as an impedance transformer.

\section{$\underline{\text { 3.Strcuture }}$}

The layout of the proposed LH CPW transformer on ferrite substrate is shown in Figure 1 (a). As shown, the employed LH unit cell consists of a short CPW section of length $\mathrm{t} 1$ loaded both sides by a series air gap capacitor of length ta and a shunt 3 arms meandered line inductor. The detailed meandered line inductor is shown in Figure 1 (b). Two feeding short CPW TLs sections of length 11 are added at both ends.

The employed ferrite substrate is the commercial Trans Tech G-113* with thickness 1 $\mathrm{mm}$. Its electric and magnetic material parameters are, dielectric constant $\varepsilon_{\mathrm{f}}=15$, dielectric loss tangent $<0.0002$, saturation magnetization $4 \pi \mathrm{M}_{\mathrm{S}}=1780$ Gauss, and magnetic linewidth $\Delta \mathrm{H}_{0} \leq 25$ Oe. An internal DC magnetic field $\left(\mathrm{H}_{0}\right)$ is applied to the ferrite substrate, and hence the saturation magnetization, in the shown direction.

The initial design of the proposed transformer was based on considering the employed ferrite substrate as an isotropic one. This can be done by assuming a very high DC magnetic bias applied to the ferrite substrate. Hence, the onset cut off frequency characterizing the dispersive properties of the hosting ferrite CPW TL is shifted to a very frequency. Hence, the ferrite substrate can be characterized only with its electric properties within the transformer operating frequency. Under this condition, the proposed ferrite $\mathrm{LH}$ transformer was designed to match a $200 \Omega$ load $\left(\mathrm{Z}_{\text {load }}\right)$ to $50 \Omega$ transmission line $\left(\mathrm{Z}_{\mathrm{ol}}\right)$ at frequency $3.45 \mathrm{GHz}$. Accordingly, the LH TL should have $100 \Omega$ characteristic impedance $\left(\mathrm{Z}_{\mathrm{LH}}\right),\left(\mathrm{Z}_{\mathrm{LH}}=\left(\mathrm{Z}_{\mathrm{ol}} * \mathrm{Zl}_{\mathrm{oad}}\right)^{0.5}\right)$, and $+90^{\circ}$ phase shift at its 
output terminal. These two conditions were fulfilled by the proper selection of both loading and hosting CPW TL elements.

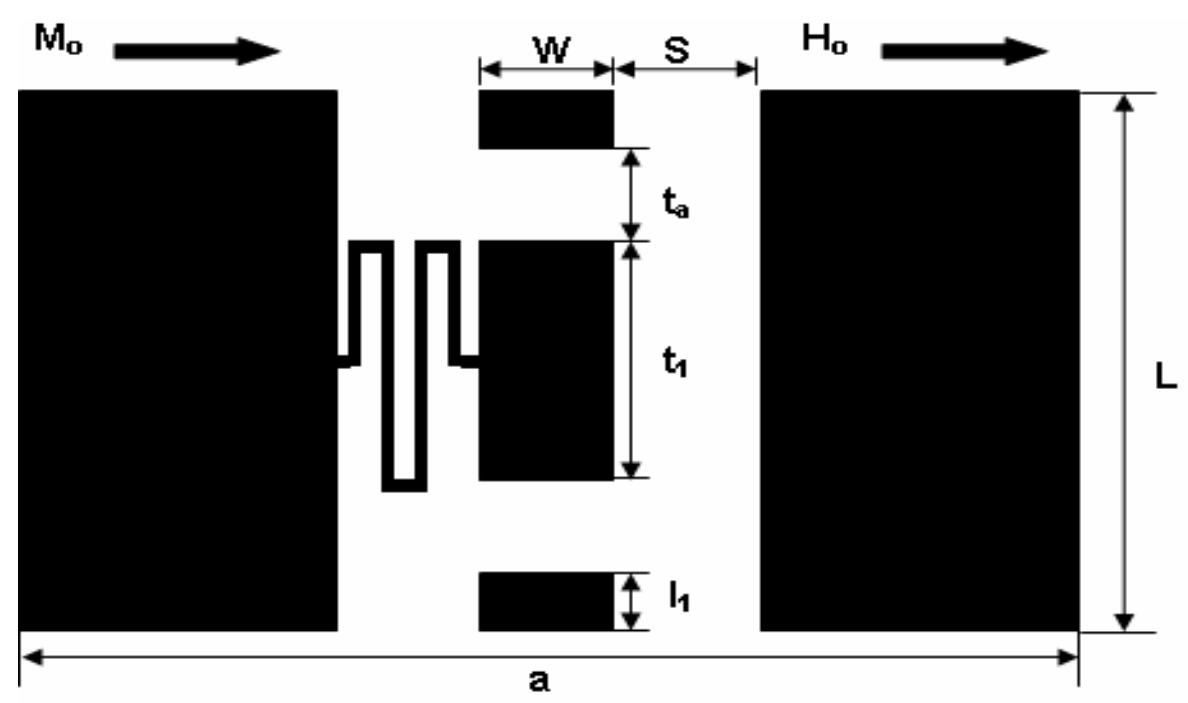

(a)

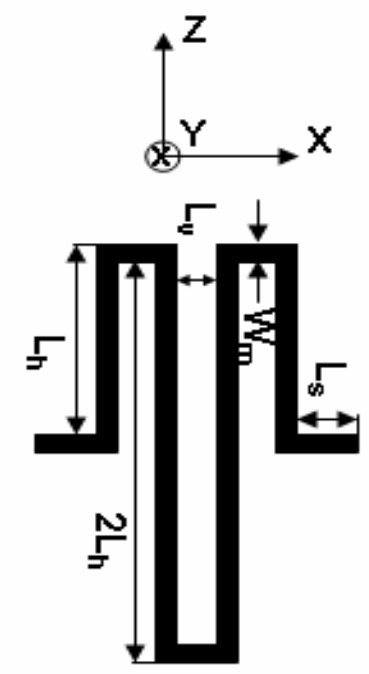

(b)

Figure (1): (a) The layout of the ferrite LH CPW high impedance transformer a $=32.75$ $\mathrm{mm}, L=2.4 \mathrm{~mm}, W=1.8 \mathrm{~mm}, t_{1}=0.4 \mathrm{~mm}, t_{a}=0.5 \mathrm{~mm}, l_{l}=0.5 \mathrm{~mm}(\mathrm{~b})$ The geometry of meander line inductor $W_{m}=0.05 \mathrm{~mm}, L_{s}=0.357 \mathrm{~mm}, L_{h}=0.6 \mathrm{~mm}, L_{v}=0.475 \mathrm{~mm}$

\section{Results}

The analytic theory of the proposed transformer was verified numerically its electromagnetic full wave simulations employing the commercial software ANSOFTHFSS. For simplicity, the applied DC magnetic field is assumed to be uniform in all studied cases.

The phase shift of the ferrite LH TL transformer by applying very high DC magnetic bias of $\mathrm{H}_{0}=50,000 \mathrm{Oe}$, is shown in Figure 2. The figure shows that the TL has a $90^{\circ}$ phase shift in the LH passband at $3.5 \mathrm{GHz}$ approximately. The return loss and the transmission loss of the transformer terminated with a $200 \Omega$ load are shown in the same figure. It is evident that the transformer has minimum return loss better than $25 \mathrm{~dB}$ with an almost $0 \mathrm{~dB}$ transmission loss at $3.5 \mathrm{GHz}$. Also, it has a $3 \mathrm{~dB}$ bandwidth from 3.05 $\mathrm{GHz}$ to $4.4 \mathrm{GHz}(36 \%)$. 


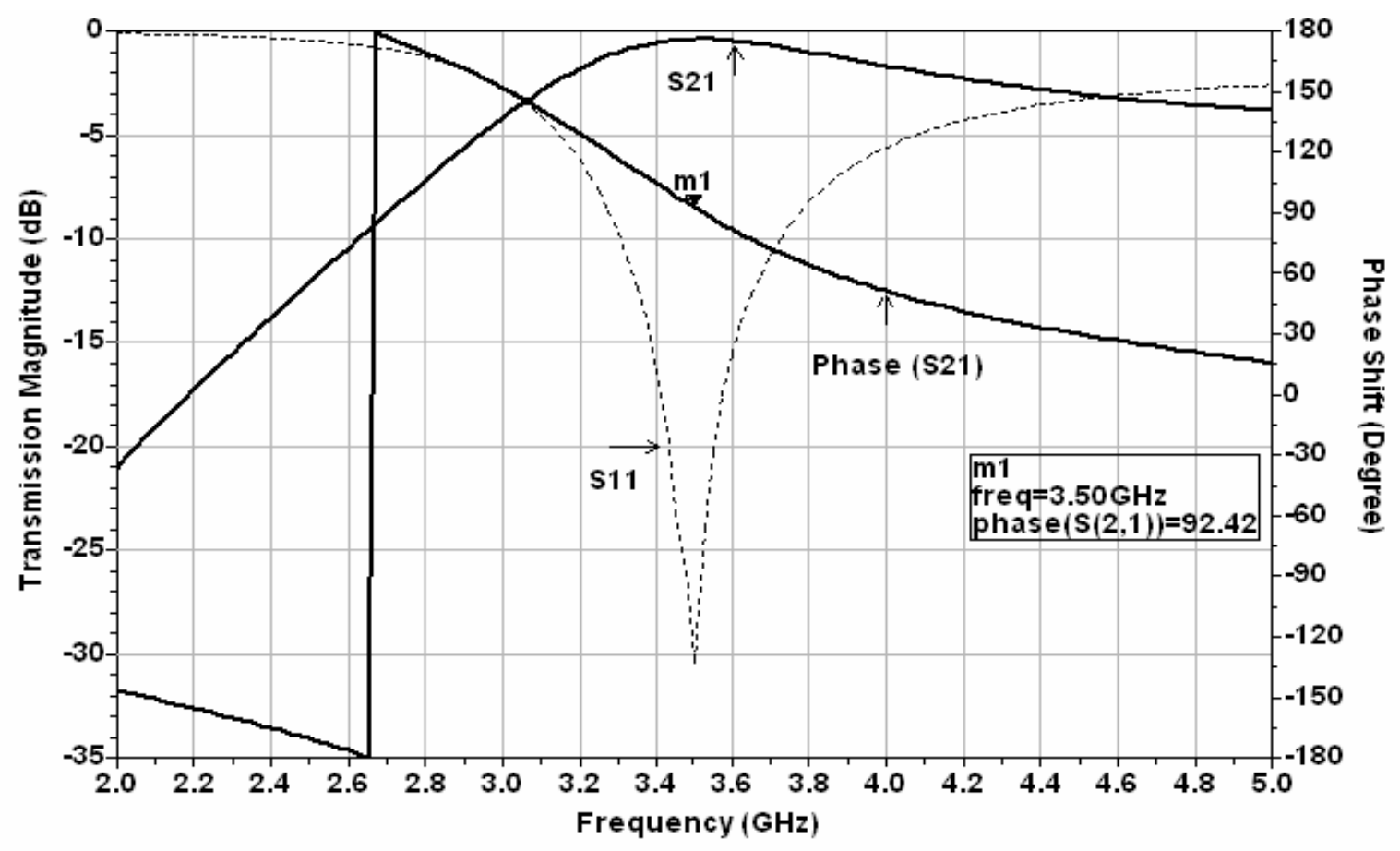

Figure (2): The full wave simulated scattering parameters (magnitudes and phase) of the high impedance ferrite transformer, $H_{0}=50,000$ Oe.

To illustrate the tuning capability of the ferrite LH transformer, two more lower DC magnetic bias cases of 1000 Oe and 3000 Oe have been numerically studied as shown in Figure 3. It is clear that the operating frequency of the transformer can be tuned from $2.8 \mathrm{GHz}$ in the first case to $3.35 \mathrm{GHz}$ in the second case with return loss better than 20 $\mathrm{dB}$ and insertion loss almost $0.5 \mathrm{~dB}$ in both cases. Also, the operating bandwidth is tuned from $2.55 \mathrm{GHz}$ to $3.1 \mathrm{GHz}(20 \%)$ in the first case, to be from $2.95 \mathrm{GHz}$ to 4.2 $\mathrm{GHz}(35 \%)$ in second case.

By further increasing the DC applied bias, the tunability of the transformer becomes clearer. The change of centre frequency of the transformer against the DC bias is shown in Figure 4. As shown in the figure, the transformer centre frequency has non linear variation with the applied DC magnetic bias. It increase from $2.8 \mathrm{GHz}$ at $\mathrm{H} 0=1000 \mathrm{Oe}$ to $4 \mathrm{GHZ}$ at $\mathrm{H0}=1500 \mathrm{Oe}$ and decreases to $3.05 \mathrm{GHz}$ at $2000 \mathrm{Oe}$. Then, this increase decrease process repeats two times with small frequency variations steps. Finally, the matching frequency reaches $3.4 \mathrm{GHz}$ at $4500 \mathrm{Oe}$ and continues constantly up to 5000 Oe. 


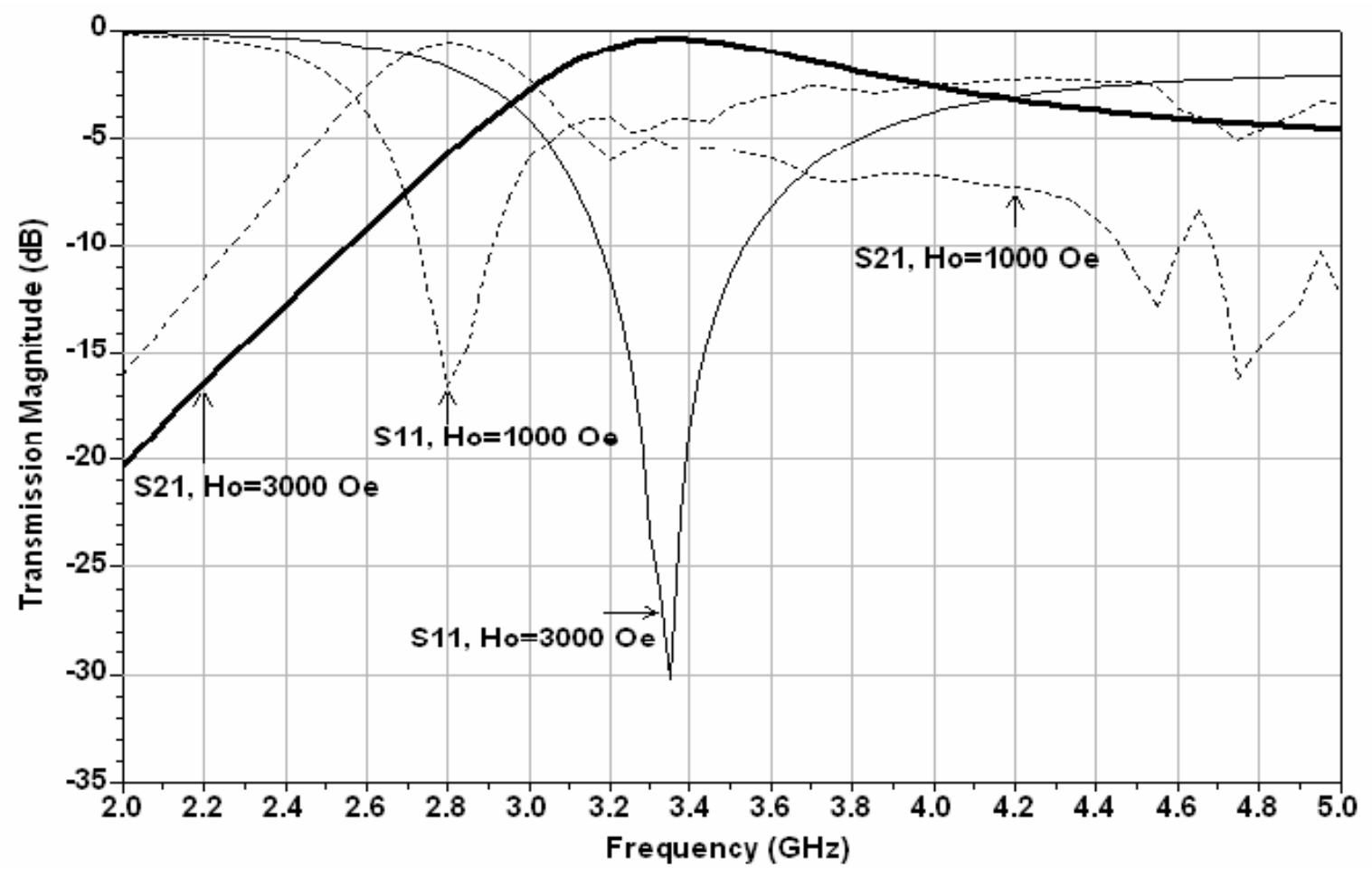

Figure (3): The full wave scattering parameters magnitude of the proposed transformer for $\mathrm{H}_{0}=1000$ and $3000 \mathrm{Oe}$

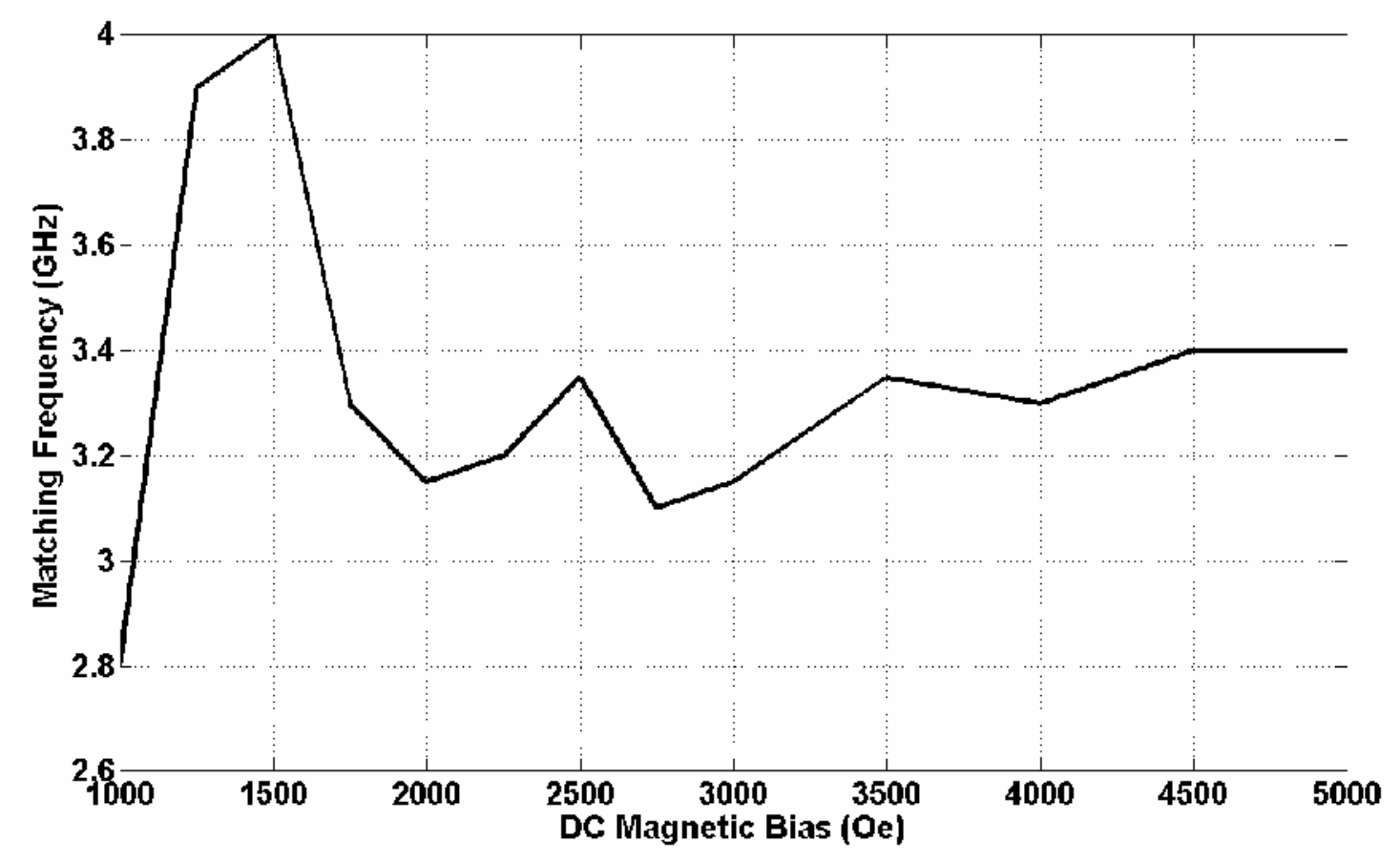

Figure (4): Variation of the centre frequency against the DC magnetic bias. 


\section{Conclusions:}

A high impedance compact ferrite tunable LH transformer model has been introduced. The LH transformer was designed using simple series air gap capacitors and shunt meandered line inductor. The proposed transformer has been studied analytically and numerically. The numerical results confirm that the transformer can match a $200 \Omega$ load and a $50 \Omega$ line whose whole bandwidth can be tuned whose center frequency changes from $2.8 \mathrm{GHz}$ to $3.4 \mathrm{GHz}$ by changing the applied DC magnetic bias. The proposed transformer size is only $2.4 \mathrm{~mm}$ which is approximately $78 \%$ reduction in conventional RH transformer length operates within the same frequency band.

\section{References:}

[1] Falcone F, T Lopetegi, J D Baena, R Marques, F Martin, and M Sorolla, Effective negative- $\varepsilon$ stopband microstrip lines based on complementary split ring resonators, IEEE Microwave and Wireless Components Lett., Vol. 14, No. 6, P. 280-282, 2004.

[2] Elefteriades G V, A K Iyer, P C Kremer, Planar negative Refractive index media using planar L-C loaded transmission Line, IEEE Trans. Microwave Theory \& Tech., Vol. 50, Vo. 12, P. 2702-2712, 2002.

[3] Caloz C., T. Itoh, Transmission line approach of left-handed ( $\mathrm{LH}$ ) materials and microstrip implementation of an artificial LH transmission line, IEEE Trans. Antennas and Propagation., Vol. 52, Vo. 5, 1159-1166, 2004.

[4] J. Machae, M. Hudlieka, J. Zehentner, N. G. Spiliotis, and A. S. Omar, Lefthanded coplanar waveguide, in Proc IEEE MTT-S, 2005, P. 1957-1960.

[5] Caloz C., T. Itoh, Electromagnetic Metamaterials Transmission Line Theory and Microwave Applications, 1st ed., New jersey: John Wiey \& Sons, 2006.

[6] Eleftheriades G. V., K. G. Balmain, Negative Refractive Metamaterials, 1st ed., New jersey: John Wiey \& Sons, 2005.

[7] Lax B, K. Button J. Microwave Ferrites and Ferrimagnetics. McGraw Hill, 1962.

[8] Tsutsumi M. and T. Ueda, Nonreciprocal left-handed microstrip lines using ferrite substrate, in Proceedings of IEEE MTT-S Int. Microwave Symposium, USA, June 2004, P. 249-252.

[9] Abdalla M. and Z. Hu, On the study of cwp dual band left handed propagation with reciprocal and nonreciprocal characteristics over ferrite substrates, in Proceedings of IEEE AP-S Int. Symp, USA, June 2007, P. 2578-2581. 